\title{
Influence of the COVID-19 pandemic on Asian scholarly journal editors' daily life, work, and opinions on future journal development
}

\author{
Yeonok Chung ${ }^{1}$, Sue Kim², Sun Huh ${ }^{3}$ \\ ${ }^{1}$ Department of Social Welfare, Jangan University, Hwaseong; ${ }^{2}$ College of Nursing, Yonsei University, Seoul; ${ }^{3}$ Department of \\ Parasitology and Institute of Medical Education, College of Medicine, Hallym University, Chuncheon, Korea
}

\section{Abstract}

Purpose: This study examined changes in Asian journal editors' daily life and work during the coronavirus disease 2019 (COVID-19) pandemic and investigated their opinions on expected changes, thereby providing preliminary data to support the future needs of journal editors.

Methods: A survey questionnaire was developed and sent to 1,537 editors and staff of Asian scientific journals from July 13 to 19,2020 . The items gathered information on participants' general characteristics, changes in daily life, changes in work life, anticipated future changes, and suggestions in the context of the COVID-19 pandemic.

Results: Of the 152 respondents (response rate, 9.7\%), most were editors. Fifty-seven respondents $(37.5 \%)$ felt very or extremely anxious about the COVID-19 pandemic, and $101(68.4 \%)$ reported spending more time on the internet. The workload of editing, reviewing, and publishing had increased for about one-third of respondents $(34.2 \%, \mathrm{n}=52)$. Forty-four respondents (28.9\%) said that the number of submissions had increased. Of the 68 editors who had received manuscripts on COVID-19, 30 (44.1\%) prioritized them. Most respondents $(73.7 \%, \mathrm{n}=112)$ predicted that online-only journal publishing would expand after the COVID-19 pandemic.

Received: August 7, 2020 Accepted: August 11, 2020

Correspondence to Sun Huh shuh@hallym.ac.kr

ORCID

Yeonok Chung

https://orcid.org/0000-0003-0008-5772

Sue Kim

https://orcid.org/0000-0003-3785-2445 Sun Huh

https://orcid.org/0000-0002-8559-8640
Conclusion: COVID-19 appears to be a source of anxiety to editors, which may be related to the increased time they spend on the internet. Some editors reported an increased workload. To promote online communication, a better environment and training tools are required. Editors and staff will need more opportunities to prepare for online publishing, as editors believed that the online-only publication of scholarly journals would accelerate after the COVID-19 pandemic.

Keywords

COVID-19; Severe acute respiratory syndrome coronavirus 2; Publishing; Anxiety; Internet 


\section{Introduction}

Background/rationale: On March 11, 2020, the World Health Organization declared coronavirus disease 2019 (COVID-19) as a pandemic to enable more vigorous measures to control and prevent this viral disease. Although this infectious disease has spread worldwide, there is no specific drug or available vaccine at this point. In response to the COVID-19 pandemic, social distancing was implemented throughout the world, including Asia, as a preventive measure to limit the transmission of COVID-19 [1]. Research activities have also been affected, although the impact of countermeasures against $\mathrm{CO}$ VID-19 has varied across scientific fields. For example, the situation differs considerably between fields where research can be conducted online and those where laboratory research is required. Moreover, in response to the need for rapid exchange of current knowledge, many journals have received increased submissions [2], although the patterns in the number of submissions are not uniform [3]. Collectively, these findings suggest that journal editors may face changes in their workload.

No study has yet investigated work changes or changes in daily life among editors of scholarly journals during the COVID-19 pandemic. It may be meaningful to understand how social changes originating from this pandemic have influenced editors' daily life and work life. Furthermore, understanding journal editors' expectations regarding the future of journal publishing will provide a basis for strategies aiming to support their efforts to develop their journals.

Objectives: This study aimed to examine the changes in Asian journal editors' daily life and work life, and to gather opinions on anticipated changes in the age of the COVID-19 pandemic. The specific goals were as follows: to identify any changes in editors' daily life and work life, to determine whether aspects of editors' daily life or work life differed depending on their characteristics, to identify their opinions on directions for the development of academic journals, and to derive suggestions for supporting editors according to the above results. These results will identify current challenges that editors face and can help editors and publishers to set long-term directions for journal development in the future. Furthermore, this study will be able to generate the changes in researchers' writing activities and publishing environment.

\section{Methods}

Ethics statement: This study was approved by the institutional review board of Hallym University (HIRB-2020-037). Informed consent was obtained from respondents through an online survey.
Study design: This cross-sectional descriptive study employed an online survey.

Development of the measurement tool: The survey items were initially developed by one author (YC), and were then modified through discussions among seven executive board members of the Korean Council of Science Editors from June 1 to July 10, 2020. After expert agreement on all items, which were verified to be related to journal editors' work or environment, 19 survey items were finalized. A reliability test was not done since only two items gathered Likert-scale interval data. The other categorical (nominal) items could not be treated as binomial data; therefore, a goodness-of-fit test could not be executed. The 19 survey questions are presented in Suppl. 1. The survey consisted of four items on the general characteristics of respondents, two items on changes in daily life, seven items on changes in work life, and six items on anticipated future needs. The items on changes in daily life were rated on a 5 -point Likert-scale and there were four open-ended items on expectations regarding the future. The other 12 items were categorical.

Participants: The mailing lists of the Korean Council of Science Editors and the Korean Federation of Science and Technology Societies $(n=1,225)$ were used for recruitment, as well as the mailing list of the Council of Asian Science Editors $(\mathrm{n}=312)$. In total, 1,537 invitations to the survey were sent via email, with one follow-up reminder.

Data analysis: The frequency of each item of the survey questionnaire was calculated. A correlation analysis of the two Likert-scale items was done. The items were compared according to respondents' characteristics. To evaluate the significance of the association between an increased workload and an increased number of submissions, the chi-square test was done after simplifying the data into "yes" and "other" responses. DBSTAT ver. 5.0 (DBSTAT, Chuncheon, Korea) was used for the statistical analysis. Content analysis was conducted of the descriptive responses presenting reasons for participants' categorical responses to items related to online journal publishing, increases in the number of articles, changes following COVID-19, and suggested topics for editor training.

\section{Results}

The results of the survey are available in Dataset 1.

Participants: Out of 1,537 invited participants, 152 (9.7\%) responded from July 13 to 19,2020 . Their role in journal publishing and affiliations are tabulated in Table 1 . The majority were editors or editorial board members $(80.9 \%, n=123)$. Therefore, the results of this survey may be considered to reflect their opinions.

All respondents were from Asia, reflecting the fact that the 
Table 1. Role and affiliation of respondents

\begin{tabular}{|c|c|c|c|c|c|c|}
\hline & $\begin{array}{c}\text { Editor or editorial board } \\
\text { member }\end{array}$ & Manuscript editor & Staff, academic society & Staff, publishing company & Other & Total \\
\hline University & $110(92.4)$ & $8(6.7)$ & $1(0.8)$ & $0(0.0)$ & $0(0.0)$ & $119(100)$ \\
\hline Publishing company & $2(33.3)$ & $2(33.3)$ & $0(0.0)$ & $2(33.3)$ & $0(0.0)$ & $6(100)$ \\
\hline Academic society & $2(14.3)$ & $4(28.6)$ & $8(57.1)$ & $0(0.0)$ & $0(0.0)$ & $14(100)$ \\
\hline Subtotal & $123(80.9)$ & $17(11.2)$ & $9(5.9)$ & $2(1.3)$ & $1(0.7)$ & $152(100)$ \\
\hline
\end{tabular}

Values are presented as number (\%).

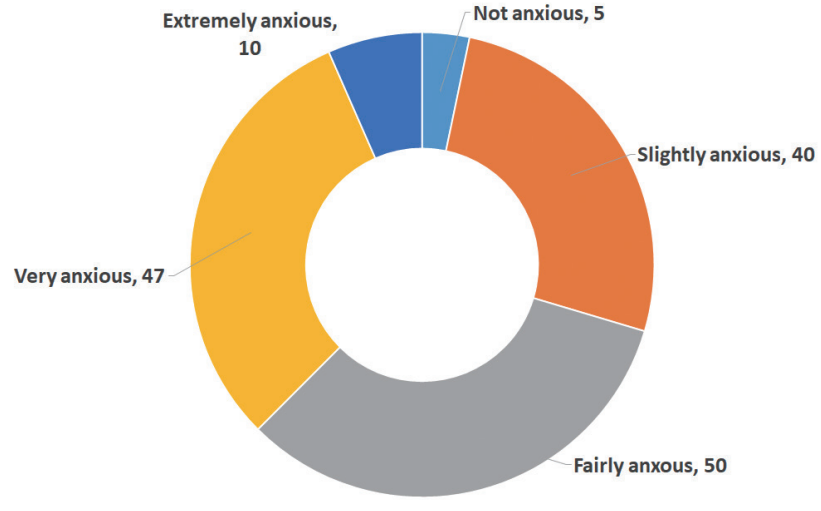

Fig. 1. Respondents' anxiety levels about the COVID-19 pandemic.

invitations were sent to the mailing list of organizations in Asia. Their research fields as follows: medical and health sciences, 65 (42.8\%); engineering, 35 (23.0\%); natural sciences, 20 (13.2\%); arts, humanities, and social sciences, 18 (11.8\%); agriculture and fisheries, 12 (7.9\%); and others, 2 (1.3\%).

Main results

Daily-life: Fifty-seven respondents (37.5\%) felt very anxious or extremely anxious about COVID-19 (Fig. 1). More than two-thirds of the respondents $(67.1 \%, \mathrm{n}=102)$ reported spending more time on the internet during the COVID-19 pandemic (Fig. 2). The correlation between anxiety level and the time spent on the internet was weak, but statistically significant $(\mathrm{r}=0.1986 ; \mathrm{P}=0.0142 ; 95 \%$ confidence interval, 0.0405 to 0.3468 ).

Work life: Eighty respondents (52.6\%) said that they worked at home or alternated between working at home and their institutional office (Fig. 3). Fifty-two respondents (34.2\%) stated that their workload increased after the COVID-19 pandemic. This response was especially common for respondents in natural sciences $(9 / 20)$. There was no association between research field and the workload excluding agriculture and fish-

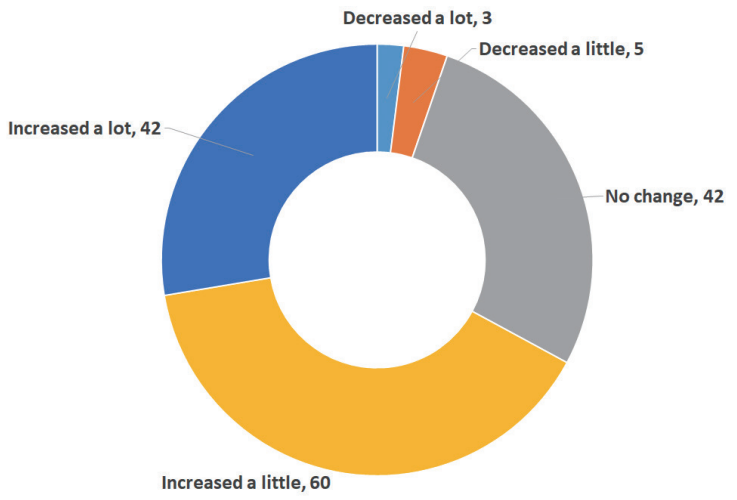

Fig. 2. Changes in time spent on the internet since the COVID-19 pandemic.

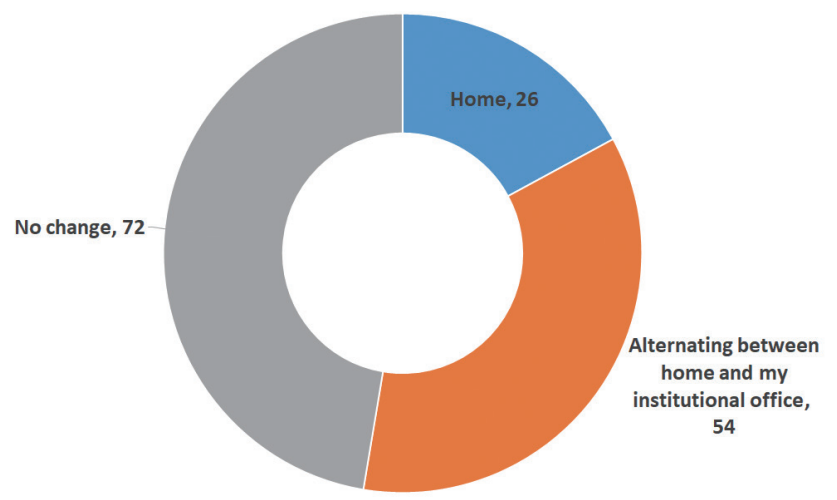

Fig. 3. Main workplace since the COVID-19 pandemic.

eries due to the response cell value less than 5 (Table 2, Fig. 4).

Forty-one respondents (27.0\%) reported difficulties in communicating with other editorial board members or publishing colleagues. Since the COVID-19 pandemic, 44 respondents $(28.9 \%)$ noted an increased number of submissions to their main journal than in previous years. The field where submissions had increased the most was the arts, humanities, and social sciences $(8 / 18,44.4 \%)$. This was followed in de- 
Table 2. Chi-square test of the association between research field and workload

\begin{tabular}{lccc}
\hline & \multicolumn{3}{c}{ Workload } \\
\cline { 2 - 4 } Field & Increased & $\begin{array}{c}\text { No change or } \\
\text { decreased }\end{array}$ & Total \\
\hline Engineering & 10 & 25 & 35 \\
Natural science & 9 & 11 & 20 \\
Medicine & 21 & 44 & 65 \\
Social science & 7 & 11 & 18 \\
Total & 47 & 91 & 138 \\
\hline
\end{tabular}

$d f=3$, significance level (alpha $=0.05): \chi^{2}=7.82, P=0.6125$.

Table 3. Chi-square test of the association between research field and number of submissions

\begin{tabular}{lccc}
\hline & \multicolumn{3}{c}{ No. of submissions } \\
\cline { 2 - 4 } Field & Increased & $\begin{array}{c}\text { No change or } \\
\text { decreased }\end{array}$ & Total \\
\hline Engineering & 5 & 25 & 30 \\
Natural science & 7 & 10 & 17 \\
Medicine & 21 & 39 & 60 \\
Social science & 8 & 10 & 18 \\
Total & 41 & 84 & 125 \\
\hline
\end{tabular}

$d f=3$, significance level (alpha $=0.05): \chi^{2}=7.82, P=0.1496$.

Table 4. Chi-square test of the association between increased workload and increased number of submissions

\begin{tabular}{lccc}
\hline $\begin{array}{l}\text { Increased } \\
\text { workload }\end{array}$ & \multicolumn{3}{c}{ Increased submissions } \\
\cline { 2 - 4 } & Yes & Others & Total \\
\hline Yes & 25 & 24 & 49 \\
Others & 19 & 69 & 88 \\
Total & 44 & 93 & 137
\end{tabular}

$d f=1$, significance level (alpha $=0.05): \chi^{2}=3.84, P=0.0008$.

scending order, by natural sciences $(7 / 20,35.0 \%)$, medical and health sciences $(21 / 65,32.3 \%)$, agriculture and fisheries $(3 / 12,25.0 \%)$, and engineering $(5 / 35,14.3 \%)$. There was also no association between the research field and the number of submissions excluding agriculture and fisheries due to the response cell value less than 5 (Table 3, Fig. 5).

Fifty-one respondents (33.6\%) said that they spent more time on reviewing or editing manuscripts since COVID-19, while 90 stated that there was no change (59.2\%) and 11 (7.12\%) noted a decreased time spent. The chi-square test showed a significant association between workload and the

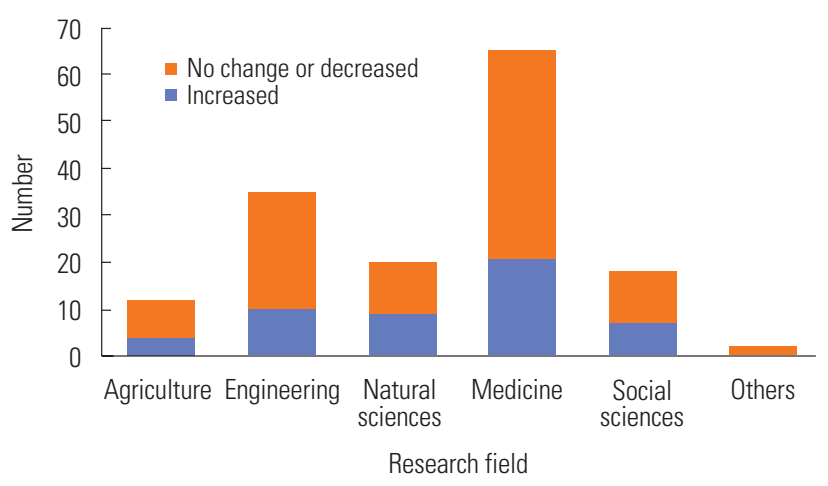

Fig. 4. Changes in editors' workload during the COVID-19 pandemic period according to the research field.

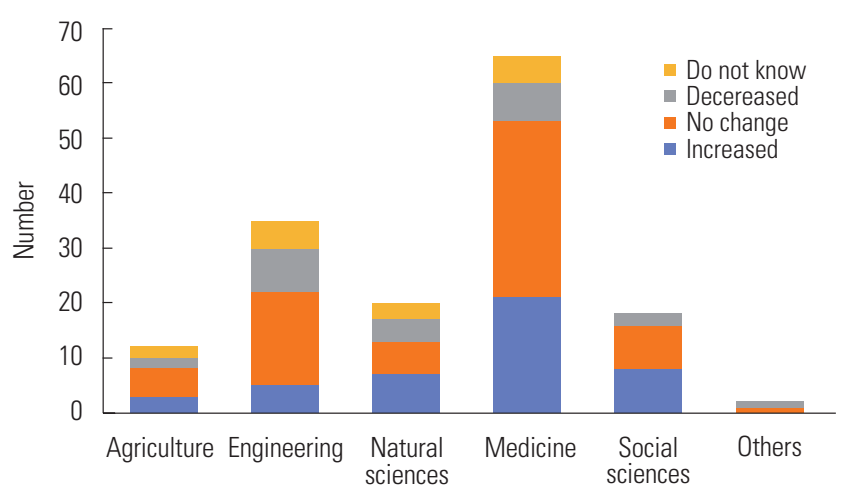

Field

Fig. 5. Changes in the number of submissions since the COVID-19 pandemic.

number of submissions. In this analysis, responses of "do not know" (15) were removed, and responses of "no change" and "decreased" items were merged as "others" (Table 4).

When editing or reviewing manuscripts related to COVID-19 (if any), 38 out of 68 editors (55.9\%) indicated that they prioritized those manuscripts (e.g., fast-track review). As for training and learning from March to June 2020, more than half of the respondents $(52.6 \%, \mathrm{n}=80)$ stated that they had not been able to attend any online training programs for journal editing or publishing (Fig. 6).

Editor's expectations for the future of journal publishing: The majority of respondents $(73.7 \%, \mathrm{n}=112)$ thought that onlineonly journal publishing would expand after the COVID-19 pandemic. The reasons can be summarized as follows (Suppl. 2): easy access (7), economic advantages (3), non-face-to-face exchange (1), rapid communication (3), and irreversible trends (38). Forty respondents stated that there would be no changes.

Sixty-four respondents (42.1\%) said that the need for jour- 


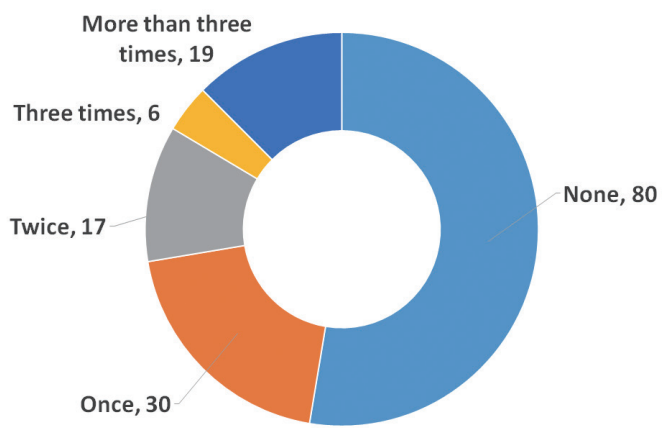

Fig. 6. Frequency of attending online training programs for journal editing and publishing from March to June 2020.

nal publishing (the number of articles or number of journals) would increase after the COVID-19 pandemic. The reasons for this anticipated increase can be summarized as follows (Suppl. 3): the emergence of new topics due to the COVID-19 pandemics (11), more time for authors to write (9), expansion of the information market (1), and the government's pressure to publish (1). In contrast, the reasons given by respondents who anticipated no changes in the number of articles included decreased research activity (including exchange programs) (10), no expected change in the publication environment (10), negative economic impacts (3), and the saturated journal market (3) (Suppl. 3).

The changes respondents expected in journal editing and publishing due to COVID-19 are tabulated in Suppl. 4. From 62 answers, the following topics were extracted: reduced submissions (7), increased submissions (1), rapid or sharp review (2), more active use of preprint servers (2), more active online publishing (12), a transition to online meetings (7), the need for an excellent editing team (3), increased budget (3), and more competition (1). Seventeen persons expected no change in the journal publishing environment.

Suggested topics for future training programs related to editing or publishing are summarized in Suppl. 5. From 43 answers, the following topics were extracted: editing or editorial process (15), publication ethics (9), upgrading the publishing process (which included the use of Open Journal Systems [https://pkp. sfu.ca/ojs/], digital standards of scholarly journals, ISO XML standards, and the journal management system) (8), online training (4), improving peer review (4), indexing in international databases (2), and editors' collaboration (1).

\section{Discussion}

Key results: One-third of the 152 respondents felt very or extremely anxious about the COVID-19 pandemic. Two-thirds of respondents spent more time on the internet during the COVID-19 period. The correlation between anxiety level and time spent on the internet was statistically significant, albeit weak. One-third of respondents reported an increased workload in editing, reviewing, and publishing. Of the editors who indicated that they received submissions related to COVID-19, nearly half prioritized such manuscripts, although it is unclear whether this may have contributed to their anxiety. Three quarters thought that online-only journal publishing would expand after the COVID-19 pandemic.

Interpretation: During the COVID-19 pandemic, it appears that a sizable proportion of editors or publishing staff experienced anxiety, as reports have also shown for the general population [4]. They also reported spending more time on the internet, which may be inevitable due to social distancing and the change in the workplace from institutional offices to home. In countries such as Korea, where social distancing and home-based working policies were implemented starting in the early phase of the COVID-19 pandemic, this was more of a social mandate than personal choice.

As editing, reviewing, and publishing scholarly journals are doable both from the office and at home, the extension of work to home may have been natural in terms of flow and results. Although weak, the correlation between anxiety and increased internet time may be worth further examination in the future. As shown in Table 4, an increased number of submissions was associated with an increased workload among editors although the majority of editors did not receive more submissions and their workload did not increase. This is understandable because editors' workload can be estimated according to the number of submissions. A previous report indicated that manuscripts on COVID-19 were processed rapidly, with a median acceptance of 6 days [2]. Although fasttrack review has some issues involving the quality of the review process, the circumstances of the COVID-19 pandemic may spur editors and reviewers to accelerate the process. Editors may want to recruit many manuscripts on COVID-19 to disseminate reliable information more rapidly to prevent disease transmission, achieve favorable treatment results, and speed vaccine development, which may also help explain the association of an increased workload with increased submissions. However, this pattern may fuel a cycle of stress and anxiety for editors and publishers, and this issue may need to be examined closely as the pandemic becomes prolonged. There was a different pattern of workload and number of submissions according to the fields (Figs. 4, 5). It could be anticipated that medical editors receive more submissions and their workload increased. However, results were different from the anticipation. Editors from natural science and the arts, humanities, and social sciences said that their workloads in- 
creased and they received more submissions although it is difficult to say there were any differences among research fields (Tables 2, 3).

Since the reason for the increased need for journal publishing included the emergence of new topics due to the COVID-19 pandemic and more available time for authors, the research articles on COVID-19 regardless of research fields may foreseeably steadily increase even after the pandemic may dissipate. Therefore, editors should be ready to receive and review the articles on COVID-19. However, reasons for no change in the number of submissions included decreased research activities, decreased exchange programs, and negative economic impact, which may also be crucial to some research fields. Thus, sufficient budget and exchange of manpower may also be needed to maintain scholarly journal output across all fields.

Finally, respondents' suggestions for training programs highlighted concrete areas that can be covered in future conferences or workshops held by editors' associations. Aside from traditional topics such as improving reviewing and editing, emerging topics included online training, and publishing technology and platforms (for example, the Open Journal System, digital standards, and ISO XML production).

Comparison with previous studies: Life changes and psychological stress during the COVID-19 pandemics among the general population were analyzed in Germany. Out of 15,704 German adults, $44.9 \%$ showed generalized anxiety regarding COVID-19 [4]. In Taiwan, an online questionnaire commenced on February 14, 2020. Of 3,555 adults, 52.1\% reported moderate to severe anxiety symptoms [5]. As such, other studies have investigated anxiety during the COVID-19 pandemic, but no previous research has specifically investigated daily life changes or changes in work among editors or publishing staff.

Limitation: The response rate was low (9.7\%), most likely due to the short survey period. Most of the individuals who were invited to respond were from Korea (79.7\%). Therefore, the results may not reflect all Asian editors' opinions. Another limitation is that anxiety was measured using a single question. Although weak, the correlation between anxiety and increased internet time may be worth further examination in the future. The meaning of internet use may be different according to the purpose of internet use. If it is mainly for editing or publishing, the anxiety level may not be associated with internet use per se, whereas searching the internet for COVID-19 information and developments and/or seeking entertainment may be associated with anxiety. In this survey, the allocated time of internet use was not inquired separately in light of this limitation, for more detailed data on editors' anxiety and further challenges, another survey or study is required.
Generalizability: Although the survey subjects were concentrated in Korea, this survey provides a quick snapshot of the present circumstances and work environment of Asian editors.

Conclusion: This survey found that the COVID-19 pandemic was a source of considerable anxiety for more than one-third of editors and publishers, especially as they spent more time on the internet. Some of them worked harder during the COVID-19 pandemic period than before and their increased workload was associated with an increased number of submissions. As such, a closer examination of editors' and publishers' work and anxiety may be needed as the pandemic drags on. While traditional topics for training programs were still valid (e.g., publication ethics, more advanced journal management, and editors' collaboration, etc.), a sizable proportion of respondents believed that more editorial or publishing work would be done online in the future. Thus, preparing editors for automated processing and educating them on digital standards appears to be an emerging area of need.

\section{Conflict of Interest}

Sun Huh has been the President of the Korean Council of Science Editors since January 17, 2020, but has no role in the decision to publish this article. Except for that, no potential conflict of interest relevant to this article was reported.

\section{Data Availability}

Dataset file is available from: the Harvard Dataverse at: https://doi.org/10.7910/DVN/XVHPUF

Dataset 1. Raw data of the survey

\section{Supplementary Material}

Supplementary file is available from the Harvard Dataverse at: https://doi.org/10.7910/DVN/XVHPUF

Suppl. 1. Survey questionnaire

Suppl. 2. Reasons for responses of "yes" to the item "Do you think that online-only journal publishing will expand after the COVID-19 pandemic?"

Suppl. 3. Reasons for responses of "yes" or "no" to the item "Do you think that the need for journal publishing (the number of articles or number of journals) will increase after the COVID-19 pandemic?"

Suppl. 4. Answers to the item "Please describe any other changes you expect in journal editing and publishing due to COVID-19."

Suppl. 5. Answers to the item "Please suggest future training programs related to editing or publishing." 


\section{References}

1. Central Disaster and Safety Countermeasure Headquarters of the Republic of Korea. Rules and guidelines for distancing in daily life to control coronavirus disease 2019 in Korea: 3rd version, announced on July 3, 2020. J Educ Eval Health Prof 2020;17:20. https://doi.org/10.3352/jeehp.2020.17.20

2. Palayew A, Norgaard O, Safreed-Harmon K, Andersen TH, Rasmussen LN, Lazarus JV. Pandemic publishing poses a new COVID-19 challenge. Nat Hum Behav 2020;4:666-9. https://doi.org/10.1038/s41562-020-0911-0

3. Flaherty C. No room of one's own: early journal submission data suggest COVID-19 is tanking women's research productivity [Internet]. Washington, DC: Inside Higher Ed; 2020 [cited 2020 Aug 7]. Available from: https://www. insidehighered.com/news/2020/04/21/early-journal-submission-data-suggest-covid-19-tanking-womens-research-productivity

4. Bauerle A, Teufel M, Musche V, et al. Increased generalized anxiety, depression and distress during the COVID-19 pandemic: a cross-sectional study in Germany. J Public Health (Oxf) 2020;fdaa106. https://doi.org/10.1093/pubmed/fdaa106

5. Wong LP, Hung CC, Alias H, Lee TS. Anxiety symptoms and preventive measures during the COVID-19 outbreak in Taiwan. BMC Psychiatry 2020;20:376. https://doi.org/10.1186/ s12888-020-02786-8 